\title{
Análisis del perfil sociodemográfico, deportivo y psicológico en una práctica de escalada deportiva de estudiantes universitarios Analysis of sociodemographic, sport and psychological profile in a rock-climbing experience on university students

\author{
Pablo Morilla Portela*, Socorro Rebollo Rico*, Antonio Baena Extremera**, María Teresa Miranda León* y \\ Manuel Alfonso Martínez Marín** \\ *Universitad de Granada, **Universidad de Murcia
}

\begin{abstract}
Resumen: La relación entre diversos factores psicológicos y la práctica de escalada ha quedado demostrada desde hace tiempo, sin embargo, la mayoría de los trabajos realizados se limitan a situaciones muy artificiales y alejadas de la naturaleza. Muy pocos son los estudios que han llevado a cabo este tipo de investigaciones en el medio natural y han combinado la recogida de datos con una práctica real de escalada. Los instrumentos utilizados para la recogida de datos han sido dos cuestionarios: el CSAI-2 y uno específicamente diseñado para recoger la información deseada sobre características sociodemográficas y hábitos deportivos. En nuestro trabajo hemos analizado diversas características de los participantes (perfiles sociodemográfico, deportivo general y deportivo como practicante de actividades físicas en el medio natural) y hemos comprobado cómo se relacionan entre ellas y su correlación con diferentes factores psicológicos (ansiedad cognitiva, ansiedad somática y autoconfianza). A través de éste artículo se evidencia como hay un porcentaje mayor de participantes mujeres que de hombres que escalan IV y V grado, mientras que en grados superiores los porcentajes se igualan. En lo referente a aspectos psicológicos, podemos apreciar como aquellos sujetos que escalan grados de mayor dificultad y que tienen un mayor interés por la práctica de la escalada, experimentan por un lado menores niveles de ansiedad somática y cognitiva y por otro, mayores niveles de autoconfianza.

Palabras clave: Escalada deportiva, ansiedad cognitiva, ansiedad somática y autoconfianza.
\end{abstract}

\begin{abstract}
The relationships among several psychological factors in rock climbing was proved a long time ago, nevertheless, most researches are limited to very artificial situations, far away from nature. There are few studies which have carried out this kind of investigation in the natural environment and have combined data collection with real rock climbing practice. The instruments used for this data collection were two questionnaires: CSAI-2 and another one specifically designed to gather the necessary information about sociodemographic characteristic and sport habits. In our work we have studied various individuals' features (sociodemographic, general sport and outdoor profiles) and we have confirmed how they are interrelated and their influence on several psychological factors (cognitive anxiety, somatic anxiety and self-confidence). Through this article we show that there are higher percentages of women than men participants who climb IV-V grade, whereas in higher grades the percentages equalize. Regarding psychological factors, we can notice how on the one hand those participants who climb higher grades and are more interested in rock climbing, feel lower cognitive anxiety and somatic anxiety, while on the other hand they feel higher self-confidence levels.
\end{abstract}

Key words: Rock climbing, cognitive anxiety, somatic anxiety and self-confidence.

\section{Introducción}

Desde hace algunos años, estamos siendo testigos de un crecimiento notable y de un auge en nuestra sociedad, por la práctica de todos aquellos deportes que tienen en común la naturaleza como terreno de práctica (Baena \& Rebollo, 2008; García, 2006; Granero \& Baena, 2007). Esto no se debe a un hecho casual, ya que la tendencia actual se orienta hacia la desvinculación forzosa del hombre con la naturaleza, y en última instancia con sus orígenes. Este suceso provoca en el ser humano un anhelo de encontrarse consigo mismo, algo que solo puede llevarse a cabo en el medio natural.

El deporte de la escalada surge de una ancestral tradición consistente en subir grandes montañas y que innumerables culturas han desarrollado. La escalada se ha convertido desde antaño, tanto en una actividadrecreativa como un deporte de élite altamente competitivo (Giles, Rhodes \& Taunton, 2006; Watts, 2004), que ha sufrido una gran evolución en las últimas décadas, fundamentalmente debido al espíritu innovador de sus practicantes y al desarrollo de los materiales. Ambos factores han permitido que desde los años 80 y principios de los 90, la escalada evolucione surgiendo nuevos estilos, que según Draper, Jones, Fryer, Hodgson y Blackwell (2010), se pueden clasificar en: de primero, donde el escalador asciende asegurando su progresión a través de anclajes en la pared; de segundo, donde el escalador asciende liberando la cuerda del dispositivo que lo fija a la pared; en top rope, donde la cuerda pasa por una reunión u otro seguro, que hay en la parte más alta de la vía; free o solo, donde el ascenso es hecho sin cuerda; y boulder, que es escalar una vía, frecuentemente corta, sin cuerda y con un compañero que asegura una posible caída.

Fecha recepción: 11-10-12 - Fecha envío revisores: 06-11-12 - Fecha de aceptación: 27-02-13 Correspondencia: Pablo Morilla Portela

C/Pintor López Mezquita, $n^{\circ}$ 9, $6 \mathrm{H}$.

18002 Granada

E-mail: pablomp@correo.ugr.es
Desde que se comenzó a investigar en torno a la escalada hasta ahora, el mayor de número de estudios se ha interesado fundamentalmente por aspectos fisiológicos del deporte (Bertuzzi, Franchini, Kokubun \& Kiss, 2007; Booth, Marino, Hill \& Gwinn, 1999; de Geus, O’Driscoll \& Meeusen, 2006; Doran \& Grace, 2000; Draper, et al., 2012; Draper \& Hodgson, 2008; Morrison \& Schöffl, 2010), y ha sido prácticamente en laúltima década, cuando ha comenzado a valorarse el papel de los factores psicológicos. Estos, debido a las características intrínsecas de este deporte, juegan un papel primordial y algunos de los mismos, han comenzado a estudiarse con más profundidad por diferentes autores (Delle Fave, Bassi \& Massimini, 2003; Ferrand \& Tetard, 2006; Hunt \& Daines, 2004; Llewellyn, Sánchez, Asghar \& Jones, 2008; Llewelyn \& Sánchez, 2008; Martha, Sánchez \& Gomà-iFreixanet, 2009; Robinson, 1985; Sánchez, Boschker \& Llewellyn, 2010; Sánchez, Lambert, Jones \& Llewellyn, 2010).

Boyd y Munroe (2003) examinaron en un doble estudio, por un lado hasta qué punto los escaladores principiantes y expertos usaban las cinco funciones de la imaginería, y por otro, cómo los practicantes de este deporte se diferenciaban de deportistas que se dedicaban al atletismo en el uso de esta técnica.

Pijpers, Oudejans, Holsheimer y Bakker (2003), también realizaron un doble estudio para analizar las manifestaciones de la ansiedad en escaladores a diferentes niveles: subjetivo, fisiológico y comportamental. En el primero, un grupo de escaladores debía llevar a cabo dos rutas transversales idénticas en un rocódromo. La única diferencia entre las vías es que una de ellas está a .3 metros del suelo y la otra a 5.1 metros. Los participantes sometidos a esta prueba manifestaron niveles de ansiedad superiores escalando la vía que se encontraba a mayor altura. En su segundo estudio, Pijpers et al. (2003), se basa en la literatura previa sobre ansiedad (Hardy, 1999; Masters, 1992; Mullen \& Hardy, 2000) argumentando que ésta podría tener como consecuencia en el escalador, movimientos menos ágiles y más torpes y tensos. Para ello, determinaron la fluidez de los movimientos midiendo la entropía. Los 
resultados revelaron como los escaladores obtenían peores resultados en la vía de escalada que se encontraba a mayor altura. Estos datos apoyaron su hipótesis inicial en la cual un aumento en los niveles de ansiedad, asociados a un incremento de tensión muscular, mayor tiempo de escalada e incremento en la entropía producían unos patrones de movimiento menos eficaces.

En el trabajo de Hardy y Hutchinson (2007) se realizaron varias investigaciones a la vez. En la primera manipularon la ansiedad cognitiva estudiando los niveles alcanzados mediante el test $R C A I$, desarrollado por Hardy y Whitehead (1984). Los participantes escalaron dos vías, una al límite de su dificultad y otra, dos grados por debajo. Los resultados confirmaron que los escaladores experimentaban niveles más altos en la vía que estaba al límite de sus posibilidades en comparación con la vía que estaba dos grados por debajo. En la segunda parte de su investigación todos los escaladores ascendieron por vías que estaban al límite de sus posibilidades (de primero y en top-rope), comparando a aquellos que habían obtenido niveles de ansiedad cognitiva altos en el primer estudio, con aquellos que los habían obtenido bajos. Los resultados confirmaron que los participantes de ambos grupos mantenían niveles de ansiedad similares y que los sujetos experimentaban niveles de ansiedad cognitiva mayores durante la escalada de primero que durante la escalada en toprope. En su última parte del estudio la comparación fue hecha entre los niveles de ansiedad cognitiva de escaladores que ascendían (siempre al límite de sus posibilidades) una misma vía dos veces, de primero y en top-rope, y que después escalaban una segunda vía en top-rope dos veces. Similares niveles de ansiedad cognitiva fueron registrados cuando los escaladores se disponían a ascender vías desconocidas, independientemente de que el sujeto fuera de primero o en top-rope. El ascenso de primero se caracterizó por niveles significativos en la ansiedad somática.

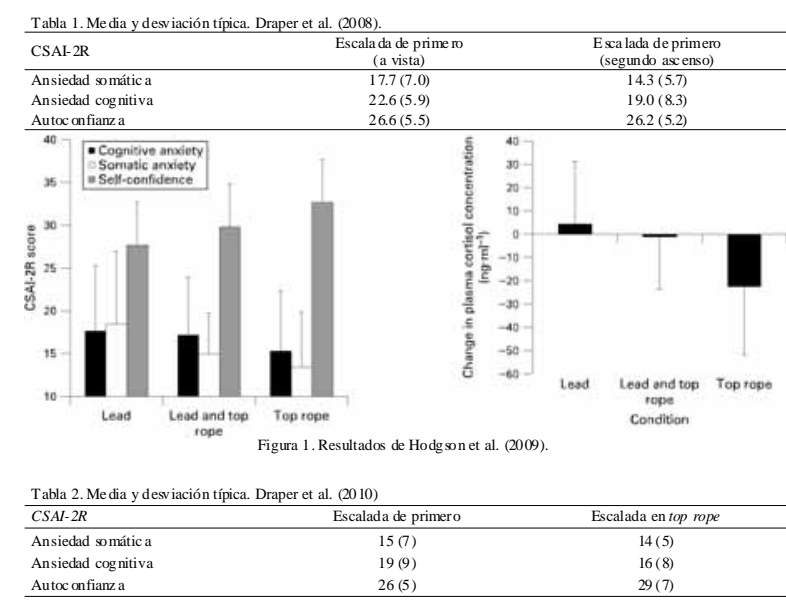

Draper, Jones, Fryer, Hodgson y Blackwell (2008), demostraron la importancia de los aspectos psicológicos en escalada deportiva para escaladores con un nivel intermedio, evidenciando los cambios que se producían en la ansiedad experimentada cuando subían una vía a vista, en comparación con el segundo ascenso que efectuaban a la misma vía (Tabla 1). Estos autores encontraron diferencias significativas en los resultados del CSAI-2R para la ansiedad cognitiva y somática, pero no para la autoconfianza.

En relación con la habilidad de los escaladores para evaluar la seguridad percibida Martha, Sánchez y Gomà-i-Freixanet (2009), comprobaron que el riesgo percibido por los escaladores se corresponde fielmente con el riesgo al que se exponen y voluntariamente asumen.

Otro trabajo como el de Hodgson, Draper, McMorris, Jones Fryer y Coleman (2009), demuestran como diferentes tipos de escalada, provocan diferentes estados de ansiedad somática, ansiedad cognitiva y autoconfianza(Figura 1).Además, la investigación evidencia como existe una relación entre la ansiedad somática, ansiedad cognitiva autoconfianza y las concentraciones de cortisol en plasma.

Draper et al. (2010), estudiaron las diferencias fisiológicas y psicológicas en 9 escaladores de nivel intermedio, teniendo en cuenta los diferentes tipos de escalada y evaluando justo antes de la actividad la ansiedad de los escaladores que participaron en el estudio. En los datos de laTabla2, se muestra como no se encontraron resultados significativos en lo que a la ansiedad se refiere.

Teniendo en cuenta todo lo anterior, en el presente estudio nos planteamos como objetivo analizar el perfil sociodemográfico, deportivo y psicológico y a establecer si existe algún tipo de relación entre ellos.

\section{Método}

\section{Muestra}

La selección de la muestra fue de carácter no probabilístico y por conveniencia, siendo un total de 280 alumnos matriculados en la asignatura «Actividades Físicas en el Medio Natural», impartida en curso el $5^{\circ}$ curso de la titulación de Licenciado en Ciencias de laActividad Física y del Deporte, en la Facultad de Ciencias de la Actividad Física (FCCAFD) de la Universidad de Granada (España).

Los mayores porcentajes de alumnos se encontraban comprendidos entre las edades de veintiuno a veintitrés años. En cuanto al sexo y a la situación laboral, podemos observar por un lado como existe una mayor proporción de hombres (72\%) frente a las mujeres (28\%), y por otro como el 69\% son estudiantes (no realizando ningún tipo de trabajo, el $24 \%$ estudia y también trabaja, dedicando al trabajo menos de 20 horas a la semana y el $7 \%$ estudia y trabaja dedicando al trabajo más de 20 horas a la semana.

\section{Variables}

La relación entre las variables y los indicadores empleados para nuestro estudio se detallan a continuación en la Tabla 3.

\begin{tabular}{|c|c|}
\hline Variable & Indicadores \\
\hline Perfil sociodemográfico & $\begin{array}{l}\text { Edad } \\
\text { Sexo } \\
\text { Año que cursa en la FCCAFD } \\
\text { Situación laboral }\end{array}$ \\
\hline Perfil deportivo en general & $\begin{array}{l}\text { Grado de interés por la a ctividad fisica } \\
\text { Nivel de práctica depootiva } \\
\text { Deportes practicados }\end{array}$ \\
\hline Perfil deportivo como practicane de AFMN & $\begin{array}{l}\text { Grado de interés por la A FMN } \\
\text { Nivel de práctica deportiva } \\
\text { Modalidad practicadade AFMN } \\
\text { AFMN practicadas } \\
\text { Personas con las que practica AFMN } \\
\text { Frecuencia con la que practica AFMN } \\
\text { Participación en competiciones de AFMN }\end{array}$ \\
\hline Perfil de escalad or & $\begin{array}{l}\text { Grado de interés por la escalada } \\
\text { Nivel de práctica de escalada } \\
\text { Lugar donde practica escalada } \\
\text { Personas con las que prac ctica esc alada } \\
\text { Grado de dificultad que escala avista } \\
\text { Motivos por los que practitac escaladad } \\
\text { Nivel de seg uridad que otorga al deporte de la esc alada }\end{array}$ \\
\hline Factores psicológicos & $\begin{array}{l}\text { Ansiedad cognitiva } \\
\text { Ansiedad somática } \\
\text { Autoconfianza }\end{array}$ \\
\hline
\end{tabular}

\section{Instrumento}

Como técnica de obtención de datos en nuestra investigación, hemos empleado la encuesta y como instrumento fundamental para la obtención de datos, el cuestionario. Debido a la diversidad de objetivos que hemos pretendido abarcar en esta investigación, hemos utilizado dos instrumentos: el primero (Cuestionario $n^{\circ} 1$ ) diseñado específicamente para este estudio. Conél se analiza por un lado, el perfil sociodemográfico, el perfil deportivo general, el perfil deportivo como practicante de actividad física en el medio natural y el perfil como escalador. Por otro, registramos diferentes factores que han tenido lugar durante la práctica de escalada llevada a cabo ( ${ }^{\circ}$ de vías escaladas, características de los componentes del grupo con los que se ha realizado la práctica, etc.) El segundo cuestionario fue diseñado y está destinado para medir los niveles de ansiedad cognitiva, ansiedad somática y autoconfianza, (Cuestionario n ${ }^{\circ}$ ), compuesto por el test Competitive State Anxiety Inventory (CSAI-2) de Martens, Vealey y Burton (1990).

Aunque el cuestionario tiene 136 variables, no hemos utilizado todos los datos recogidos en la investigación por la magnitud de los mismos. Las variables y los indicadores empleados pueden verse en la Tabla 3. 


\section{Análisis estadístico}

Tras la tabulación y elaboración de una base de datos, se ha llevado a cabo el análisis estadístico de los mismos. Para ello se ha utilizado el paquete de programa estadístico SPSS (Statistical Package for Social Sciences) versión 15.0.1.

En primer lugar se ha realizado un análisis descriptivo de los datos, mediante el procedimiento Frecuencias, obteniéndose para las variables categóricas las correspondientes tablas de frecuencias y porcentajes de cada modalidad. Para las variables cuantitativas tales como los niveles de ansiedad, se ha hallado los valores de las medias y desviaciones típicas. Igualmente se han realizado gráficos explicativos de las variables y aspectos más importantes en este trabajo. En segundo lugar se ha realizado el análisis inferencial. Para investigar la posible relación entre diversas variables categóricas medidas y diversas características de la práctica de deportes y Actividades Físicas en el Medio Natural (AFMN) se han realizado las correspondientes tablas de contingencia, realizándose el test Chi cuadrado o el test exacto de Fisher en caso de no cumplirse las condiciones de validez (ninguna casilla puede tener frecuencia esperada menor que 1 y no más del $20 \%$ de las casillas pueden tener frecuencias esperadas inferiores o iguales a cinco). Para estudiar la posible relación entre variables cuantitativas y/o ordinales, dado que las variables medidas no se comportan según la distribución normal se ha obtenido los coeficientes de correlación no paramétricos de Spearman así como su nivel de significación estadística (procedimiento Correlaciones).

\section{Procedimiento}

Antes de pasar el cuestionario a todos los sujetos, se llevó a cabo un estudio piloto, para comprobar la validez del cuestionario con los 10 primeros grupos, lo que correspondería a 50 sujetos. Se pasó el test (Cuestionario $\mathrm{n}^{\circ} 1$ ) en un primer momento a estos 50 alumnos y tres días más tarde se les volvió a pasar el retest (esta vez sin llevar a cabo la actividad de escalada). Podemos decir por consiguiente que hemos asegurado una medida de estabilidad, o confiabilidad por test-retest (Hernández, et al., 1991). A través de un cálculo de correlación (Rho de Spearman) se comprobó que el 100\% de los ítems analizados en test y retest eran significativos al nivel .01 .

La aplicación de los cuestionarios se llevó a cabo de forma autoadministrada, donde el investigador estuvo presente durante la recogida de los datos. El día que cada grupo tuvo que hacer la actividad se citó a los participantes dentro del Parque Natural de la Sierra de Huétor (Granada, España), en el sector «El nicho», lugar donde se hizo la práctica de escalada. Antes de que se les diera ninguna explicación, se les entregó el cuestionario y se les pidió que leyeran atentamente las instrucciones y contestaran al Cuestionario $\mathrm{n}^{\circ} 1 \mathrm{y}$ al Cuestionario $\mathrm{n}^{\circ} 2$. Cuando todos rellenaron la parte que se les había pedido entre unos 5 a 10 minutos aproximadamente, se les recogió los cuestionarios y comenzó la práctica, con una duración aproximada de 3 horas. Al comienzo de la actividad, se les explicó la forma correcta de utilizar el material y las técnicas adecuadas para asegurar y progresar durante la práctica de escalada. La práctica se dividió en 2 partes: la primera de 1 hora y la

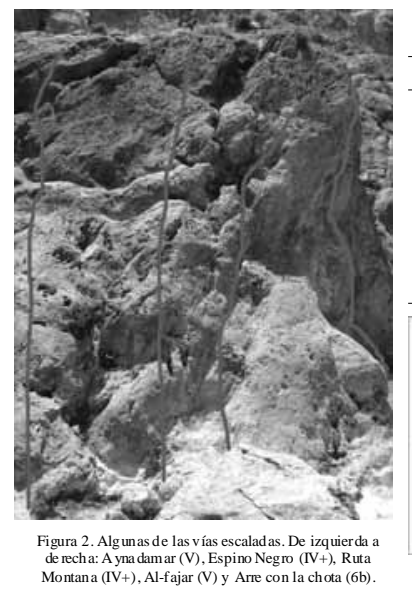

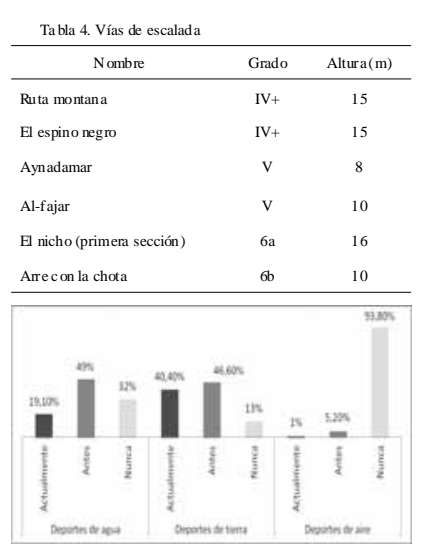

segunda de 2 horas. Durante la primera parte, la distribución de roles fue la siguiente: un asegurador, un escalador (siempre en top rope) y tres observadores. En la segunda parte de la práctica los roles desempeñados fueron los siguientes: dos aseguradores, dos escaladores y un observador. Los aseguradores siempre estuvieron supervisados y controlados por el responsable de la actividad, de forma que la seguridad de la actividad nunca dependió exclusivamente de los participantes, ni se puso en riesgo la integridad de los miembros del grupo.

Para medir el grado de escalada se utilizó la graduación internacional empleada para escalada deportiva, del mismo modo que han hecho otros autores (Draper et al., 2008). Las vías escaladas se encuentran en lazona conocida como «Escuela de escalada de Alfacar» y el número de vías utilizadas en la investigación fueron seis (Ver Tabla 4 y Figura 2).

\section{Resultados}

Una vez analizados los datos, se puede comprobar como el $78 \%$ de los sujetos afirman tener mucho interés por la práctica de actividad física y deportiva, mientras que el 22\% restante dice tener bastante interés. Estos porcentajes coinciden a la hora de responder sobre su práctica deportiva, dándose un $78 \%$ de sujetos que practica actualmente deporte y un $22 \%$ que antes si practicaba pero ahora no.

\begin{tabular}{|c|c|c|c|}
\hline Deporte & Frecue ncia de práctica & $\mathrm{N}$ & \% válido \\
\hline \multirow{3}{*}{ Deportes de pista } & Practico actualmente & 160 & 613 \\
\hline & Antes s, ahora no & 75 & 287 \\
\hline & Nunca he practicado & 26 & 10 \\
\hline \multirow{4}{*}{ Atletismo } & Practico actualmente & 30 & 115 \\
\hline & Antes s, ahora no & $\pi$ & 29.6 \\
\hline & Nunca he practicado & 153 & 588 \\
\hline & Practico actualmente & 63 & 247 \\
\hline \multirow[t]{2}{*}{ Natación } & Antes s, ahora no & 125 & 49 \\
\hline & Nunca he practicado & 67 & 263 \\
\hline \multirow{4}{*}{$\begin{array}{l}\text { G imnasia (Artísica, acr osport, } \\
\text { fitness, etc.) }\end{array}$} & Practico actualmente & 45 & $17 \mathrm{~A}$ \\
\hline & Antes si, ahora no & 58 & 225 \\
\hline & Nunca he practicado & 155 & 60.1 \\
\hline & Practico actualmente & 13 & 5.1 \\
\hline \multirow[t]{3}{*}{ Yoga, tai chi, etc. } & Antes si, ahora no & 17 & 6.6 \\
\hline & Nunca he practicado & 227 & 883 \\
\hline & Practico actualmente & 69 & 266 \\
\hline \multirow[t]{3}{*}{ Deportes de raqueta } & Antes $\mathrm{s}$, ahora no & 114 & 44 \\
\hline & Nunca he practicado & 76 & 293 \\
\hline & Practico actualmente & 34 & 13.1 \\
\hline \multirow[t]{3}{*}{ Deportes de Lucha } & Antes s, ahora no & 73 & 28.1 \\
\hline & Nunca he practicado & 153 & 588 \\
\hline & Practico actualmente & 61 & 232 \\
\hline \multirow[t]{2}{*}{ Otros } & Antes s, ahora no & 8 & 3 \\
\hline & Nunca he practicado & 194 & 738 \\
\hline
\end{tabular}

Como puede apreciarse en la Tabla 5, los deportes más practicados son los deportes de pista (futbol, baloncesto, voleibol, etc.) seguidos de lejos por los deportes de raqueta y natación.

De los alumnos que practican o han practicado deporte, el $44 \%$ tiene mucho interés por la práctica de AFMN, el $47 \%$ bastante interés y el $9 \%$ poco interés. Del mismo modo, el 31\% practica actualmente AFMN, el $45 \%$ ha practicado anteriormente aunque no lo hace actualmente y el 24\% nunca ha practicado. De aquellos que no han practicado nunca AFMN, el 66\% afirma que uno de los motivos es no conocerlos bien.

\begin{tabular}{|c|c|c|c|}
\hline Deporte & Frecuencia de prá ctica & $\mathrm{N}$ & \%válido \\
\hline \multirow{3}{*}{ Orientación } & Practico actualmente & 21 & 10.7 \\
\hline & Antessi, ahora no & 124 & 62.9 \\
\hline & Nunca he practicado & 52 & \\
\hline \multirow{4}{*}{$\begin{array}{l}\text { Trekking, carrera por } \\
\text { montaña, etc. }\end{array}$} & Practico actualmente & 18 & 9.1 \\
\hline & Antessi, ahora no & 51 & 25.9 \\
\hline & Nunca he practicado & 52 & 48.1 \\
\hline & Practico actualmente & 41 & 20.8 \\
\hline \multirow{3}{*}{ ВTT } & Antes si, ahor ano & 59 & 29.9 \\
\hline & Nunca he practicado & 97 & 49.2 \\
\hline & Practico actual mente & 5 & 2.5 \\
\hline \multirow[t]{3}{*}{ Alpinismo } & $\begin{array}{l}\text { Ante ssi, ahor ano } \\
\text { A no }\end{array}$ & 18 & 9.1 \\
\hline & Nunca he practicado & 174 & 88.3 \\
\hline & Practico actualmente & 4 & 2 \\
\hline \multirow[t]{3}{*}{ Espeleologìa } & Ante ssi, ahora no & 16 & 8.1 \\
\hline & Nunca he practicado & 177 & 89.8 \\
\hline & Practico actualmente & 75 & 38.1 \\
\hline \multirow[t]{3}{*}{ Esqui, sno wb oard, etc. } & Antessi, ahor ano & 70 & 35.5 \\
\hline & Nunca he practicado & 52 & 26.4 \\
\hline & Practico actualmente & 30 & 15.2 \\
\hline \multirow[t]{3}{*}{ De sce nso de barr ancos } & Antes si, ahora no & 97 & 49.2 \\
\hline & Nunca he practicado & 70 & 35.5 \\
\hline & Practico actualmente & 11 & 5.6 \\
\hline \multirow{2}{*}{ Raffing, ka yac, etc. } & Antessi, ahor a no & 69 & 35 \\
\hline & 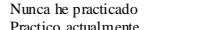 & 117 & 59.4 \\
\hline \multirow{2}{*}{ Otros } & & & \\
\hline & $\begin{array}{l}\text { Ante ssi, ahora no } \\
\text { Nunca he practicado }\end{array}$ & $\begin{array}{l}17 \\
161\end{array}$ & $\begin{array}{l}8.7 \\
82.6\end{array}$ \\
\hline
\end{tabular}


De aquellos alumnos que practican o han practicado AFMN, los mayores porcentajes en cuanto a práctica actual los encontramos en los deportes de tierra como se aprecia en la Figura 3.

Las AFMN más practicadas actualmente entre los alumnos son los deportes denieve (esquí, snowboard, etc.), BTT, descenso de barrancos y orientación (Tabla 6).

Los sujetos optan por practicar AFMN con amigos en un 90\%, con un monitor $52 \%$, con un familiar $29 \%$, con un club $16 \%$ y solos $20 \%$.

Los mayores porcentajes de práctica se concentran en los periodos festivos (40\%) en lo que se refiere a la práctica actual de AFMN y laborales y fines de semana (37\%) para aquellos que han practicado AFMN anteriormente pero no de forma actual. De estos practicantes en AFMN, el 4\% participa en competiciones actualmente y el 14\% lo ha hecho antes pero no ahora. De todos aquellos que han participado alguna vez en competiciones el $3 \%$ lo ha hecho en escalada.

El interés y la práctica de escalada pueden verse reflejados en las Figuras 4 y 5 respectivamente.

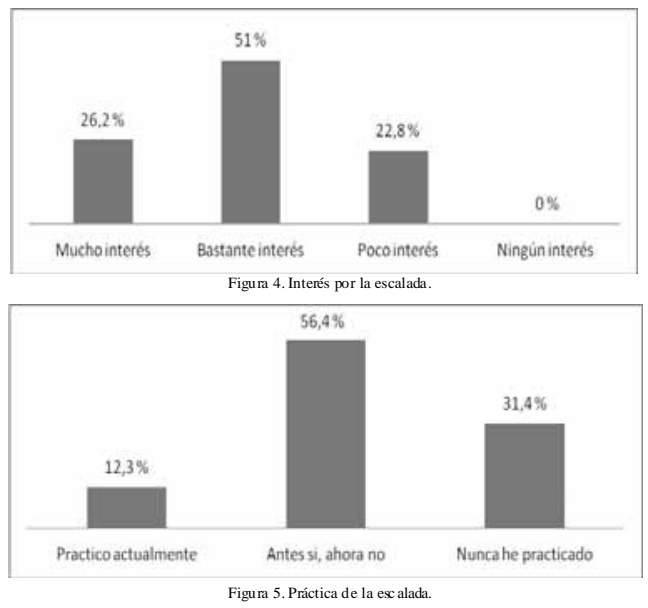

El 90\% de los encuestados que practican escalada lo hace en roca, el 63\% lo hace en rocódromo y el 19\% hace boulder o bloque. La mayoría practica escalada con un monitor o un amigo, siendo el porcentaje en ambos casos un $76 \%$. El grado de dificultad que son capaces de escalar a vista es IV-V en el 13\%, 6a-6b-6c en el 7\% y tan solo un 1\% que escala entre 7a-7b-7c.

De entre los motivos por los que practica escalada, el más seleccionado es la «Aventura personal» (ver Figura 6), y el 63\% de los participantes opina que la escalada es un deporte bastante seguro.

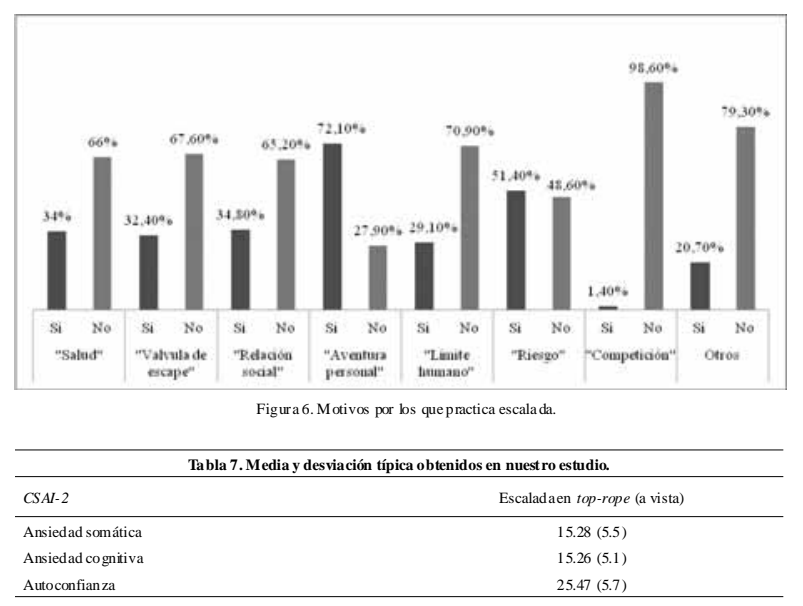

A través del CSAI-2, se obtuvieron los siguientes resultados en cuanto a ansiedad somática, ansiedad cognitiva y autoconfianza (ver Tabla 7).

Relación entre el perfil sociodemográfico y el perfil deportivo en AFMN:
Los resultados evidencian una relación inferencial positiva entre la situación laboral y la práctica de AFMN, de manera que aquellos sujetos que pertenecen a la condición de estudiante parecen practicar AFMN en menor medida, mientras que aquellos que estudian y trabajan más de 20 horas semanales lo hacen en mayor medida (ver Tabla 8).

\begin{tabular}{|c|c|c|c|c|c|c|}
\hline & & & \multicolumn{3}{|c|}{ Practica de AFMN } & \multirow{2}{*}{ Total } \\
\hline & & & Actua Imente & $\begin{array}{l}\text { Antes si, } \\
\text { ahora no }\end{array}$ & $\begin{array}{l}\text { Nunca ha } \\
\text { practicado }\end{array}$ & \\
\hline \multirow{9}{*}{$\begin{array}{l}\text { Situac ión } \\
\text { laboral }\end{array}$} & \multirow{3}{*}{ Estudiante } & $\mathrm{N}$ & 45 & 88 & 48 & 181 \\
\hline & & $\begin{array}{c}\text { \% Situación } \\
\text { laboral }\end{array}$ & $249 \%$ & $48.6 \%$ & $26.5 \%$ & $100 \%$ \\
\hline & & $\begin{array}{c}\% \text { Practica } \\
\text { AFMN }\end{array}$ & $549 \%$ & $75.9 \%$ & $77.4 \%$ & $69.6 \%$ \\
\hline & \multirow{3}{*}{$\begin{array}{l}\text { Estudiay trabaja } \\
\text { (menon de 20 horas } \\
\text { se manales) }\end{array}$} & $\mathrm{N}$ & 26 & 26 & 10 & 62 \\
\hline & & $\begin{array}{l}\text { \% Situación } \\
\text { laboral }\end{array}$ & $41.9 \%$ & $41.9 \%$ & $16.1 \%$ & $100 \%$ \\
\hline & & $\begin{array}{l}\text { \% Practica } \\
\text { AFMN }\end{array}$ & $31.7 \%$ & $22.4 \%$ & $16.1 \%$ & $23.8 \%$ \\
\hline & \multirow{3}{*}{$\begin{array}{l}\text { Estudiay trab aja (más } \\
\text { de } 20 \text { horas } \\
\text { se manales) }\end{array}$} & $\mathrm{N}$ & 11 & 2 & 4 & 17 \\
\hline & & $\begin{array}{l}\text { \% Situación } \\
\text { laboral }\end{array}$ & $64.7 \%$ & $11.8 \%$ & $23.5 \%$ & $100 \%$ \\
\hline & & $\begin{array}{l}\text { \% Practica } \\
\text { AFMN }\end{array}$ & $13.4 \%$ & $1.7 \%$ & $6.5 \%$ & $6.5 \%$ \\
\hline \multirow{3}{*}{ Total } & & $\mathrm{N}$ & 82 & 116 & 62 & 260 \\
\hline & & $\begin{array}{c}\text { \% Situación } \\
\text { laboral }\end{array}$ & $31.5 \%$ & $44.6 \%$ & $23.8 \%$ & $100 \%$ \\
\hline & & $\begin{array}{l}\text { \% Practica } \\
\text { AFMN }\end{array}$ & $100 \%$ & $100 \%$ & $100 \%$ & $100 \%$ \\
\hline
\end{tabular}

Se demuestra a través de los resultados de nuestro estudio (ver Tabla 9) cómo hay una relación inferencial entre el sexo y el grado de escalada que los sujetos son capaces de superar a vista. Se observa un mayor número de mujeres y menor número de hombres que escalan IVV grado, mientras que en niveles más exigentes (6a-6b-6c y 7a-7b-7c) están igualados. Entre los participantes que no saben o no contestan hay una mayor presencia de hombres y menor de mujeres.

\begin{tabular}{|c|c|c|c|c|c|c|}
\hline & & & \multicolumn{3}{|c|}{ Grado de escalada } & \multirow[t]{2}{*}{ Total } \\
\hline & & & IV-V & $6 a-7 c$ & No sabe/No contesta & \\
\hline \multirow{6}{*}{ Sexo } & & Recue nto & 7 & 7 & 74 & 88 \\
\hline & Hombre & $\%$ Sexo & $8.0 \%$ & $8.0 \%$ & $84.1 \%$ & $100 \%$ \\
\hline & \multirow{4}{*}{ Mujer } & $\%$ Grado de escalada & $412 \%$ & $700 \%$ & $74.7 \%$ & $698 \%$ \\
\hline & & Recue nto & 10 & 3 & 25 & 38 \\
\hline & & $\%$ Sexo & $263 \%$ & $7.9 \%$ & $65.8 \%$ & $100 \%$ \\
\hline & & \% Grado de escalada & $588 \%$ & $30.0 \%$ & $25.3 \%$ & $302 \%$ \\
\hline \multirow{3}{*}{\multicolumn{2}{|c|}{ Total }} & Recue nto & 17 & 10 & 99 & 126 \\
\hline & & $\%$ Sexo & $135 \%$ & $7.9 \%$ & $78.6 \%$ & $100 \%$ \\
\hline & & $\%$ Grado de escalada & $100 \%$ & $100 \%$ & $100 \%$ & $100 \%$ \\
\hline
\end{tabular}

Chi-cuadr ado de Pearson: Valor 7.763. Significación 21. 1 casilla (16.7\%) tiene una frecuenciae sperada inferior a 5.

Se evidencia también como el grado de dificultad que los sujetos son capaces de superar a vista tiene una relación significativa con la ansiedad cognitiva, la ansiedad somática y la autoconfianza por un lado. Los sujetos que son capaces de escalar a vista grados de dificultad más altos

\begin{tabular}{|c|c|c|c|c|}
\hline Rho de Sperman & & $\begin{array}{l}\text { Ansiedad } \\
\text { cogniitiva }\end{array}$ & $\begin{array}{l}\text { Ansiedad } \\
\text { somática }\end{array}$ & Autoc on fianz a \\
\hline \multirow{3}{*}{$\begin{array}{l}\text { Gra do de dificultad } \\
\text { escalado a vista }\end{array}$} & $\begin{array}{l}\text { Coeficiente de } \\
\text { correlación }\end{array}$ & $-2511^{(* *)}$ & $-.291(* *)$ & $.300(* *)$ \\
\hline & Sig. (bilatera l) & .005 & .001 & . 001 \\
\hline & $\mathrm{N}$ & 125 & 125 & 124 \\
\hline \multicolumn{5}{|c|}{$\begin{array}{l}\text { *Lac or elación es significativa al nivel.05 (bilateral). } \\
\text { ** La correlación es significativa al nivel } .01 \text { (bilateral). }\end{array}$} \\
\hline \multicolumn{5}{|c|}{$\begin{array}{l}\text { Tabla 11. Correlaciones bivariad as Rho de Spearman de las var iables interés por la escalada, ansiedad cognitiva, } \\
\text { somátic ay autoconfianza. }\end{array}$} \\
\hline Rhode Sperman & & $\begin{array}{l}\text { Ansiedad } \\
\text { cognitiva }\end{array}$ & $\begin{array}{l}\text { Ansiedad } \\
\text { somática }\end{array}$ & Autoconfianza \\
\hline \multirow[t]{3}{*}{ Interés por la escah da } & Coeficiente de cor relación & $-.138\left(^{*}\right)$ & $-.142(*)$ & $250(* *)$ \\
\hline & Sig. (bilateral) & .050 & . 043 & .000 \\
\hline & $\mathrm{N}$ & 204 & 204 & 202 \\
\hline
\end{tabular}


presentan niveles más bajos de ansiedad cognitiva, ansiedad somática y autoconfianza (ver Tabla 10).

El interés por la escalada también ha quedado demostrado ser un factor relacionado con los niveles de ansiedad cognitiva, ansiedad somática y autoconfianza. Mayores niveles de interés por el deporte de la escalada hacen experimentar a los alumnos menores niveles de ansiedad cognitiva y somática y mayores niveles de autoconfianza (ver Tabla 11).

\section{Discusión}

El objetivo de esta investigación ha sido analizar el perfil sociodemográfico, deportivo y psicológico y a establecer si existe algún tipo de relación entre ellos. Como no existen trabajos exactamente igual a este, se ha tenido que utilizar investigaciones de diversa índole para poder discutir los resultados aquí encontrados.

Así, en relación al estudio sobre el perfil sociodemográfico y deportivo de los raiders españoles, los cuales también practican escalada, Baena (2008) concluye que el «deporte salud» y el «deporte aventura personal» son los principales motivos por los que practican este deporte. Coinciden con los sujetos de nuestro trabajo que practican escalada, los cuales en su mayoría lo hacen por el «deporte salud» $\mathrm{y}$ «deporte riesgo». Baena (2008) también informa de que las AFMN más practicadas por estos deportistas son: el trekking, la BTT, la escalada y rapel y la orientación. De los alumnos encuestados que practican actualmente escalada, los mayores porcentajes de otros deportes practicados fueron esquí o snowboard, descenso de barrancos, BTT y trekking o carrera de montaña.

Feher, Meyers y Skellys (1998) se interesaron por estudiar el perfil psicológico de los escaladores sin encontrar diferencias significativas en el estado o rasgo de los escaladores analizando los resultados según el género o el nivel de habilidad percibida a la hora de escalar.

Relacionando nuestros datos con la mencionada investigación de Pijpers et al. (2003) no podemos apoyar la afirmación que se deduce de su estudio. En nuestro trabajo, hay una mayor proporción de chicas que de chicos que escalan a vista entre los grados IV a 7c, esto debería estar asociado a movimientos más ágiles y menos torpes y tensos, que a su vez estarían relacionados con niveles de ansiedad inferiores, y que sin embargo no se registran. Tras estudiar los diferentes niveles de ansiedad, a pesar de esta mayor proporción de chicas que escalan entre los grados IV a 7c, podemos comprobar que no existen diferencias significativas en función del sexo.

Sánchez y Torregrosa (2005), conscientes de la importancia de los factores psicológicos en el desarrollo de la actividad deportiva hacen un esfuerzo en su estudio por determinar qué factores psicológicos influyen en el rendimiento del escalador. Presentan diferentes aspectos destacados por los entrevistados al hablar sobre el papel de los procesos psicológicos: procesos básicos de captación y procesamiento de información (anticipación, atención-(re)concentración, memorización e imaginería); mecanismos emocionales (estrés, gestión de la activación y gestión del riesgo); y aspectos motivacionales (autoeficacia, autoconfianza y motivación). Vemos como coincidiendo con nuestros resultados y de acorde con los autores previos, el interés por la escalada está relacionada en el rendimiento deportivo a través de la ansiedad.

Aunque el binomio escalada-ansiedad ha interesado desde hace mucho tiempo a escaladores y científicos, es difícil encontrar textos científicos con más de unas décadas de antigüedad, exceptuando a algunos pioneros en esta área como Robinson (1985), quien intuyó y posteriormente demostró como la ansiedad rasgo es menor aquellos individuos que practican escalada.

Refiriéndonos al trabajo de Hardy y Hutchinson (2007), podemos decir que estamos de acuerdo con ellos ya que en nuestra investigación queda demostrado como ante las mismas vías, aquellos escaladores que son capaces de escalar grados de dificultad más elevados a vista, presentaron niveles más bajos de ansiedad y más altos de autoconfianza.

Si comparamos nuestros resultados con los obtenidos por Draper et al. (2008) podemos observar como la ansiedad somática es menor en nuestro estudio, 15.28 (5.5), cuando la comparamos con la de aquellos sujetos que han subido de primero a vista, 17.7 (7.0), y mayor en relación a aquellos que han subido de primero en su segundo ascenso, 14.3 (5.7). Sin embargo, la ansiedad cognitiva en nuestro estudio, 15.26 (5.1), es menor en ambos casos, 22.6 (5.9) en escalada de primero a vista y 19.0 (8.3) en escalada de primero en el segundo ascenso. La autoconfianza parece ser algo menor también en nuestro estudio, 25.47 (5.7), comparada con los dos casos anteriores, 26.6 (5.5) en escalada de primero a vista y 26.2 (5.2) en escalada de primero en el segundo ascenso.

El hecho de que los sujetos de nuestro estudio presenten menores niveles de ansiedad cognitiva (ansiedad asociada a un componente psicológico, actuando sobre los elementos del pensamiento), puede deberse a que están escalando en top-rope, modalidad en la que el escalador no debe sufrir una caída importante. Estos niveles de ansiedad cognitiva son inferiores cuando se escala en top-rope, si los comparamos con una escalada de primero, tanto a vista, como en su segundo ascenso. Esta ansiedad cognitiva se presenta más baja cuando la escalada se realiza en top-rope, incluso comparando un grupo de estudiantes universitarios (en su mayoría deportistas) con un grupo de escaladores. Del mismo modo, los niveles de ansiedad somática inferiores en nuestro estudio se deben a que la escalada en top-rope genera unos niveles de ansiedad somáticos más bajos que la escalada de primero a vista, como veremos que se demuestra más adelante. Sin embargo, el obtener en nuestro estudio, por un lado, niveles de ansiedad somática inferiores a los de una escalada de primero a vista, y por otro, superiores comparados con la escalada de primero en el segundo ascenso, podría indicar que la ansiedad somática se ve menos relacionada con la modalidad de escalada (primero o top-rope) que la ansiedad cognitiva. Los niveles de autoconfianza algo inferiores en nuestro estudio se podrían explicar por contar con una población de deportistas que no están especializados en la escalada.

Los resultados obtenidos por Pijpers, Oudejans y Bakker (2005) y Pijpers, Oudejans, Bakker, y Beek(2006), en relación al comportamiento motor, fueron corroborados por Nieuwenhuys, Pijpers, Oudejans y Bakker (2008), quienes manipularon la ansiedad recreando dos vías de escalada a alturas diferentes. Aunque los escaladores eran capaces de completar las ambas vías en la que estaba a mayor altura y experimentaban mayor nivel de ansiedad, necesitaron tiempos más largos y se evidenciaron mayores tiempos agarrando las mismas presas y tuvieron que realizar un mayor número de movimientos. Este suceso se relaciona con lo defendido anteriormente. Una mayor sensación de seguridad a la hora de realizar una actividad de escalada, (tanto por estar a menor altura, como por estar escalando en una modalidad con menor riesgo) se acaba relacionando con niveles de ansiedad más elevados.

En el mismo estudio, Nieuwenhuys et al. (2008), también estudia la relación entre la ansiedad y el comportamiento visual, relacionando situaciones de mayor ansiedad con mayores tiempo de fijación de la mirada.

En lo que se refiere a los resultados obtenidos en el estudio de Hodgson et al. (2009) en el CSAI-2R, los valores obtenidos para la escalada en top rope son similares a los nuestros para la ansiedad cognitiva y algo menores para la ansiedad somática. Los escaladores de dicho estudio tienen unos niveles de autoconfianza bastante más altos. Esto podría deberse a que nuestro sujetos no son en su mayoría escaladores (aunque hay que recordar que en su mayoría son deportistas de otras modalidades). Para la escalada en top rope con las maniobras de la escalada de primero, los niveles de autoconfianza siguen siendo superiores. La ansiedad cognitiva es algo mayor y la ansiedad somática es muy similar. Por último, para la prueba de escalada de primero, aunque la autoconfianza sigue siendo superior a la que ofrecen nuestros datos, la ansiedad cognitiva y somática son superiores.

En un último estudio de Draper et al. (2010), vemos como la ansiedad somática de los escaladores que suben de primero, 15(7), o en top-rope, 14 (5), no varía mucho de los resultados que nuestros sujetos experimentan, 15.28 (5.5) (ver Tabla 12 y Tabla 13). 


\begin{tabular}{|c|c|c|}
\hline$\overline{C S A I-2 R}$ & Esca lada deprimero & Escalada en top rope \\
\hline Ansiedad somática & $15(7)$ & $14(5)$ \\
\hline Ansiedad cog nitiva & 19 (9) & $16(8)$ \\
\hline Autoc onfianz a & 26 (5) & $29(7)$ \\
\hline \multicolumn{3}{|c|}{ Tabla 13. Media y desviac ión típica obtenidos en nuestro estudio. } \\
\hline 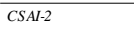 & \multicolumn{2}{|c|}{ Escalada en top-rope (a vista) } \\
\hline Ansiedad so mática & \multicolumn{2}{|c|}{$15.28(5.5)$} \\
\hline Ansiedad cog nitiva & \multicolumn{2}{|c|}{$15.26(5.1)$} \\
\hline Autoc onfianz a & \multicolumn{2}{|c|}{$25.47(5.7)$} \\
\hline
\end{tabular}

Parece ser que la autoconfianza de los 9 escaladores al subir en top rope, 29 (7), es mayor que la de nuestros sujetos, 25.47 (5.7). Por último, en cuanto a la ansiedad cognitiva vemos como para ambos casos, de primero, 19 (9) y en top rope, 16 (8), los escaladores de nuestro estudio obtienen niveles más bajos, 25.47 (5.7). El hecho de que los practicantes de escalada muestren niveles de ansiedad más altos que otro grupo formado por deportistas de diferentes modalidades deportivas puede parecer desconcertante, cuando lo normal sería que el grupo de escaladores reflejara niveles inferiores de ansiedad y superiores de autoconfianza. Creemos que el hecho de que nuestros participantes experimenten niveles menores de ansiedad puede deberse a otros motivos, los cuales intentaremos explicar en trabajos posteriores.

\section{Conclusiones}

La mayoría de los alumnos estudiados tienen bastante interés por la práctica de AFMN y han practicado con anterioridad este tipo de deportes. De los que practican o han practicado AFMN, la mayoría realiza actualmente deportes de tierra.

Las AFMN más practicadas entre los alumnos son los deportes de nieve (esquí, snowboard, etc.), la BTT, el descenso de barrancos y la orientación; la inmensa mayoría practica AFMN con amigos en periodos festivos y solo un pequeño porcentaje participa actualmente en competiciones, siendo los deportes preferidos orientación y BTT.

En cuanto a la práctica del deporte de la escalada, la mayoría de los alumnos tiene bastante interés por la escalada y ha practicado con anterioridad aunque no practique actualmente. De aquellos que practican o han practicado escalada, eligieron la escalada en roca, como el lugar más frecuente seguido del rocódromo, habiendo practicado escalada con un amigo o con un monitor en su mayoría. El motivo más seleccionado por los participantes para practicar escalada era la «aventura personal» y, en general, opinaban que era un deporte bastante seguro.

Las AFMN son practicadas en mayor medida por aquellos sujetos que estudian y trabajan más de 20 horas a la semana, mientras que son menos practicadas por aquellos que son solamente estudiantes.

Aunque tanto los hombres como mujeres que han participado en nuestra investigación, parecen estar igualados en grados de dificultad altos (6a-7c), hay mayor número de mujeres que de hombres que escalan niveles de dificultad inferiores (IV-V) y un mayor número de hombres que no sabe/no contesta el grado que es capaz de escalar.

A medida que los sujetos de nuestro estudio escalan mayores grados de dificultad, los niveles de ansiedad cognitiva y somática son menores y los niveles de autoconfianza son mayores.

Parece ocurrir algo similar con el interés por la escalada. A mayores niveles de interés por la escalada, menores niveles de ansiedad cognitiva y somática y mayores niveles de autoconfianza.

\section{Agradecimientos}

Deformageneral, queremos dar las gracias a todos aquellos estudiantes que participaron voluntariamente en la investigación, por dedicarnos su tiempo y esfuerzo, así como a nuestras familias.

A nivel particular, Pablo Morilla Portela agradece y dedica este trabajo a sus abuelos, Carmen y Rafael, y a sus padres, Emilio y Emilia, de los cuales ha recibido siempre un apoyo incondicional.

\section{Referencias}

Baena, A. (2008). Análisis del perfil sociodemográfico y deportivo de los competidores de raid de raid de aventura. Tesis doctoral. Universidad de Granada.

Baena,A., \& Rebollo, S. (2008). Análisis del perfil sociodemográfico de la mujer como participante en raids de aventura. Retos: nuevas tendencias en educación física, deporte y recreación, 14, 48-53.

Bertuzzi, R. C., Franchini, E., Kokubun, E., \& Kiss, M. A. (2007). Energy system contributions in indoor rock climbing. European Journal of Applied Physiology, 101, 293-300.

Booth, J., Marino, F., Hill, C., \& Gwuinn, T. (1999). Energy cost of sport sport rock climbing in elite performers. British Journal of Sport Medicine, 33, 14-18.

Boyd, J., y Munroe K. J. (2003). The use of imagery in climbing. Journal of Sport Pshychology, 5(2), 15-30.

De Geus, B., Villanueva O’Driscoll, S., \& Meeusen, R. (2006). Influence of climbing style on physiological responses during indoor rock climbing on routes with the same dificulty. European Journal of Applied Physiology, 98, 489-496.

Delle Fave,A., Bassi, M., \& Massimini, F. (2003). Quality of experience and risk perception in high-altitude rock climbing. Journal of Applied Sport Psychology, 15, 82-98.

Draper, N., Dickson, T., Fryer, S., Blackwell, G., Winter, D., ... \& Ellis, G (2012). Plasma cortisol concentrations and perceived anxiety in response to on-sight rock climbing. International Journal of Sports Medicine, 33, 13-17.

Draper, N., \& Hodgson, C. (2008). Adventure sport physiology. Chichester, UK: Wiley.

Draper, N., Jones, G. A., Fryer, S., Hodgson, C. I., \& Blackwell, G. (2008). Effect of an on-sight lead on the physiological and psychological responses to rock climbing. Journal of Sport Science and Medicine, 7, 492-498.

Draper, N., Jones, G. A., Fryer, S., Hodgson, C. I., \& Blackwell, G. (2010). Physiological responses to lead and top rope climbing for intermediate rock climbers. European Journal of Sport Science, 10: 1, 13-20.

Erickson, B. (2005). Style matters: explorations of bodies, whiteness, and identity in tock climbing. Sociology of Sport Journal, 22, 373396.

Feher, P., Meyers, M. C., \& Skelly, W.A. (1998) Psychological profile of rock climbers: state and trait attributes. Journal of Sport Behaviour, 21(2).

Ferrand, C., \& Tetard, S. (2006). Self-handicapping in rock climbing: A qualitative approach. Journal of Sport Applied Psychology, 18, 271-280.

García, M. (2006). Veinticinco años de análisis del comportamiento deportivo de la población española (1980-2005). Revista Internacional de Sociología, 44, 15-38.

Giles, L. V., Rhodes, E. C., \& Taunton, J. E. (2006). The physiology of rock climbing. Sport Medicine (Auckland, NZ), 36, 529-545.

Gomez López, M., Ruiz Juan, F., \& García Montes., M. E. (2005) Disponible en http://www.efdeportes.com/efd83/tlibre.htm

Granero, A., \& Baena,A. (2007). Importancia de los valores educativos de las actividades físicas en la naturaleza. Revista Habilidad Motriz, 29, 5-14.

Gutiérrez-Dávila, M., \& Oña, A. (2005). Metodología en las ciencias 
del deporte. Madrid, España: Editorial Síntesis.

Hardy, L. (1999). Stress, anxiety and performance. Journal of Science and Medicine in Sport, 2, 227-233.

Hardy, L., \& Hutchinson, A. (2007). Effects of performance anxiety on effor and performance in rock climbing: A test of processing efficiency theory. Anxiety, Stress and Coping, 20(2), 147-161.

Hardy, L., y Whitehead, R. (1984). Specific modes od anxiety and arousal. Current Psychologiacl Research and Reviews, 3, 14-24.

Hodgson, C. I., Draper, N., McMorris, T., Jones, G., Fryer, S., \& Coleman, I. (2009). Percieved anxiety and plasma cortisol concentrations following rock climbing with different safety rope protocols. British Journal of Sports Medicine, 43, 531-535.

Hunt, P., \& Brian D. (2004). Defensive processes enacted through mountaineering and their impact on climbers. British Journal of Psychotherapy, 20(4), 441-452.

Llewellyn, D. J., Sánchez, X., Asghar, A., \& Jones, G. (2008). Selfefficacy, risk taking and performance in rock climbing. Personality and Individual Differences, 45, 75-81.

Llewellyn, D. J., \& Sánchez, X. (2008). Individual differences and risk taking in rock climbing. Psychology of Sport and Exercise, 9, 413426.

Martens, R (1977). Sport Competition Axiety Test. Champaign, Illinois: Human Kinetics.

Martens, R., Vealey, R. S., \& Burton, D. (1990). Competitive anxiety in sport. Champaign, Illinois: Human Kinetics Publishers, Inc.

Masters, R. S. W. (1992). Knowledge, knerves and know-how: the role of explicit versus implicit knowledge in the breakdown of a complex motor skill under pressure. British Journal of Psychology, 83, 343358.

Martha, C., Sánchez, X., \& Gomà-i-Freixanet, M. (2009). Risk perception as a function of risk exposure amongst rock climbers. Psychology of Sport and Exercise, 10, 193-200.

Morrison, A. B., \& Schöffl, V. R. (2010). Physiological responses to rock climbing in young climbers. British Journal of Sport Medicine, $41,852-861$.

Mullen, R., \& Hardy, L. (2000). State anxiety and motor performance: testing the conscious processing hypothesis. Journal of Sports Sciences, 18, 785-799.

Nieuwenhuys, A., Pijpers, J. R., Oudejans, R. R. D., \& Bakker, F. C. (2008). The influence of anxiety on visual attention in climbing. Journal of Sport \& Exercise Psychology, 30, 171-185.

Pijpers, J. R., Oudejans, R. D., Holsheimer, F., \& Bakker, F. C. (2003) Anxiety-performance relationships in climbing: a process-oriented approach. Psychology of Sport and Exercise, 4, 283-304.

Robinson, D.W. (1985). Stress seeking: selected behavioral characteristic of Elite Rock Climbers. Journal of Sport Pshychology, 7, 400-404.

Sánchez, X., Boschker, M. S. J., \& Llewellyn, D. J. (2010). Preperformance psychological states and performance in an elite climbing competition. Scandinavian Journal of Medicine \& Sport Science in Sport, 20, 356-363.

Sánchez, X., Lambert, Ph., Jones, J., \& Llewellyn, D. J. (2010). Efficacy of pre-ascent climbing route visual inspection in indoor sport climbing. Scandinavian Journal of Medicine \& Sport Science in Sport, 20, 356-363.

Sánchez, X., \& Torregosa, M. (2005). El papel de los factores psicológicos en la escalada deportiva: un análisis cualitativo. Revista de Psicología del Deporte, 24(2), 177-194.

Pezzulo, G., Barca, L., Bocconi, A. L., \& Borghi, A. M. (2010). When affordances climb into your mind: advantages of motor simulation in a memory task performed by novice and expert rock climbers. Brain and Cognition, 73, 68-73.

Pijpers, J. R., Oudejans, R. R. D., \& Bakker, F. C. (2005). Anxietyinduced changes in movement behavior during the execution of a complex whole-body task. The Quaterly Journal of Experimental Psychology, 58A, 421-445.

Pijpers, J. R., Oudejans, R.R. D., Bakker, F. C., \& Beek, P. J. (2006). Therole of anxiety in perceiving and realizing affordances. Ecological psychology, 18, 131-161.

Watts, P. B. (2004). Physiology of difficult rock climb. European Journal of Applied Physiology, 91, 361-372.

West, A., \& Allin, L. (2010). Chancing your arm: the meaning of risk in rock climbing. Sport in Society, 13-7, 1234-1248.

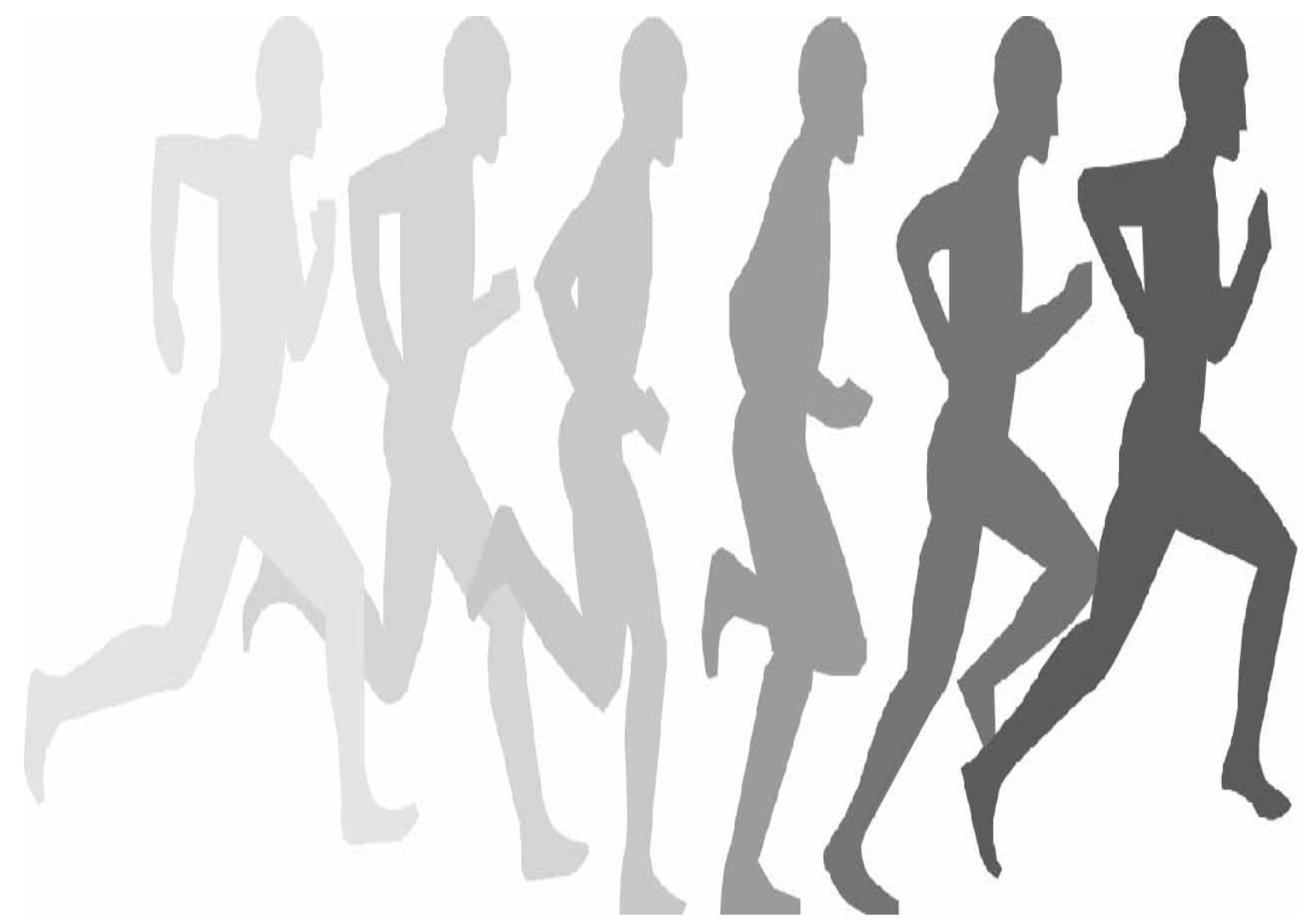

